\title{
Advanced Electron Microscopy Characterization of Intergranular Corrosion in Ni- 20Cr Alloy Under Molten Salt Environment
}

Yang Yang ${ }^{1}$, Weiyue Zhou ${ }^{2}$, Sarah Wang ${ }^{3}$, Sheng Yin ${ }^{3}$, Mark Asta ${ }^{3}, \mathrm{Ju} \mathrm{Li}^{2}$, Michael Short ${ }^{2}$ and Andrew Minor ${ }^{4}$

${ }^{1}$ Lawrence Berkeley National Laboratory, Berkeley, California, United States, ${ }^{2}$ Massachusetts Institute of Technology, Cambridge, Massachusetts, United States, ${ }^{3}$ University of California, Berkeley, Berkeley, California, United States, ${ }^{4}$ UC Berkeley and LBNL, Berkeley, California, United States

Molten salt reactors (MSR) are one of the next generation nuclear reactors that could enable much higher safety and economy with reduced radioactive waste. MSR utilizes a special coolant, i.e., molten salt, working at temperatures as high as $700^{\circ} \mathrm{C}$. While such coolant has been shown to have multiple benefits, the lack of understanding of the response of structural materials to simultaneous molten salt corrosion and radiation damage has limited materials selection, research and development and the licensing of the nuclear reactors. Here we report advanced microscopy techniques to characterize materials after molten salt corrosion with and without concurrent proton irradiation. We discovered that proton irradiation could slow down intergranular corrosion under certain situations [1].

A dedicated facility [2] that enables concurrent proton irradiation and molten salt corrosion has been constructed at MIT. With this facility, we prepared two batch of samples: (1) corrosion only; (2) concurrent corrosion and proton irradiation. Ni-20Cr alloy was chosen as a model system, while LiF-NaF$\mathrm{KF}(\mathrm{FLiNaK})$ at $650^{\circ} \mathrm{C}$ is chosen as the molten salt environment. Scanning electron microscopy (SEM) images shown that intergranular corrosion is the dominant degradation mechanism in both samples. Statistical analysis of SEM images shown that intergranular corrosion in the region without proton irradiation is more severe than that under proton irradiation. Focused ion beam (FIB) lift-out technique was used to prepare electron-transparent at selected regions for transmission electron microscopy (TEM) imaging. Ni enrichment and $\mathrm{Cr}$ depletion are found in both samples by energydispersive $\mathrm{x}$-ray spectroscopy (EDX) analysis. FIB-SEM based 3D tomography was used to probe the three-dimensional structural degradation in the samples. Four-dimensional scanning electron microscopy (4D-STEM) was applied to analyze strain around the corrosion pits. In this presentation, we will try to address several fundamental questions of the detailed mechanisms of transport in the grain-boundary regions, and how they are affected by nonequilibrium point-defect concentrations, strain and microstructures [3]. 


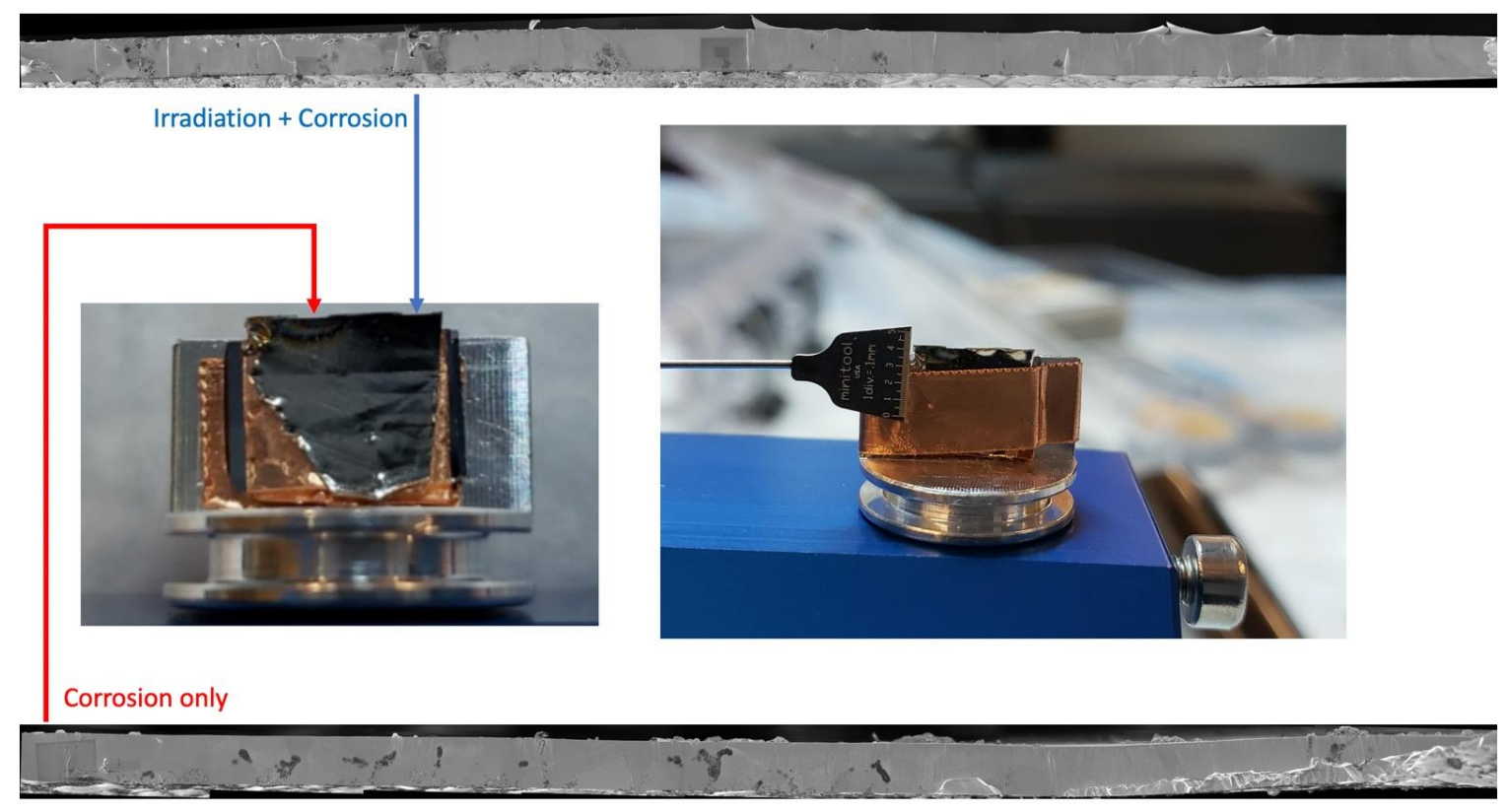

Figure 1. SEM images showing the cross-section of the samples under different corrosion conditions.
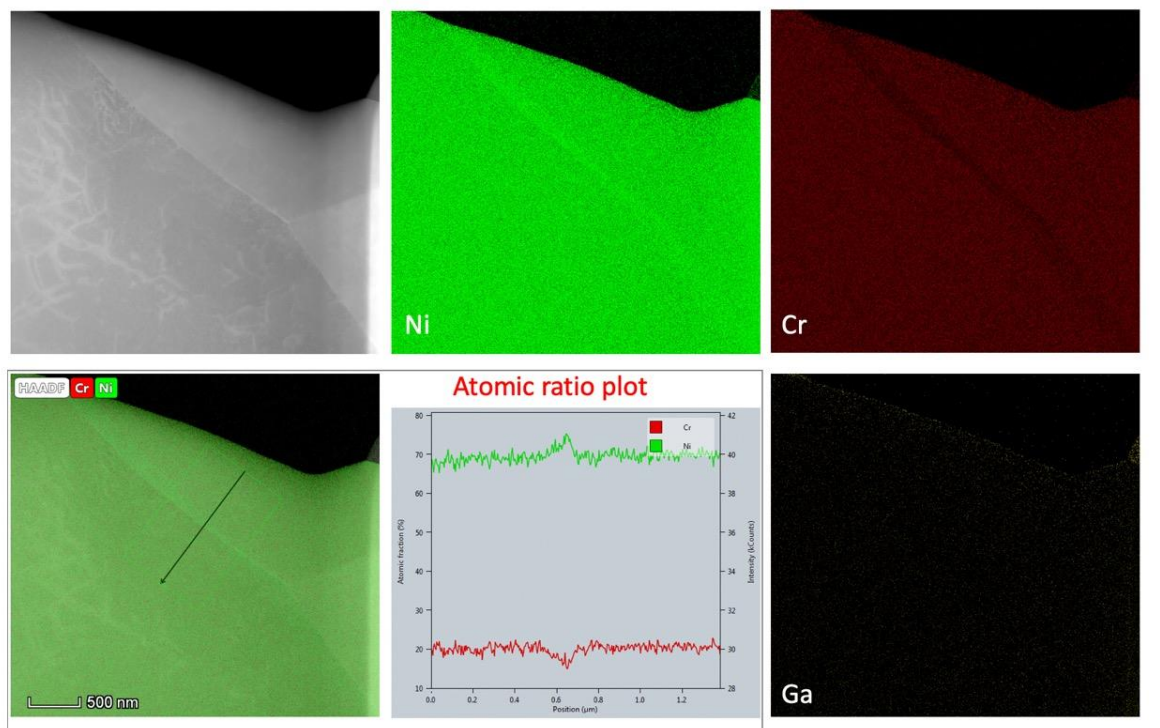

Mapping line 1 on GB 1 (close to pit): showing GB segregation

Figure 2. TEM-EDX mapping of samples showing $\mathrm{Ni}$ enrichment and $\mathrm{Cr}$ depletion at grain boundary after molten salt corrosion.

\section{References}

[1] W. Zhou, Y. Yang, G. Zheng, K. Woller, P. Stahle, A. Minor, M.P. Short, Proton IrradiationDecelerated Intergranular Corrosion of Ni-Cr Alloys in Molten Salt, Arxiv. 1911.11798 (2019). https://arxiv.org/abs/1911.11798v1.

[2] W. Zhou, K.B. Woller, G. (Tony) Zheng, P.W. Stahle, M.P. Short, A simultaneous corrosion/irradiation facility for testing molten salt-facing materials, Nucl. Instruments Methods Phys. Res. Sect. B Beam Interact. with Mater. Atoms. 440 (2019) 54-59. doi:10.1016/j.nimb.2018.11.024. 
[3] The authors acknowledge support of FUTURE (Fundamental Understanding of Transport Under Reactor Extremes), an Energy Frontier Research Center funded by the U.S. Department of Energy, Office of Science, Basic Energy Sciences. Y.Y. was supported by the Director, Office of Science, Office of Basic Energy Sciences, Materials Sciences and Engineering Division, of the U.S. Department of Energy under Contract No. DE-AC02-05-CH11231 within the Mechanical Behavior of Materials (KC 13) program at the Lawrence Berkeley National Laboratory. The authors acknowledge support by the Molecular Foundry at Lawrence Berkeley National Laboratory, which is supported by the U.S. Department of Energy under Contract No. DE-AC02-05CH11231. The authors gratefully acknowledge funding from the Transatomic Power Corporation under Grant No. 023875-001, and the US Department of Energy Nuclear Energy University Program (NEUP) under Grant No. 327075-875J. 
https://doi.org/10.1017/S1431927620013707 Published online by Cambridge University Press 